هجلة كلية التربية الرياضية .... جاهعة بغداد ..... المبلد r ..... العدد ا

\title{
علاقة بعض قدرات الإدراك الهس - حركي بدقة التصويب في كرة القدم
}

للاعبي نادي أسو الرياضي من فئة المتقدهين

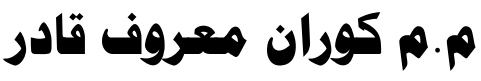

كلية التربية الرياضية - جامعة كويه

pr. q

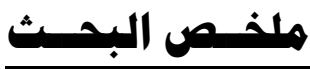

هدفت الدراسة إلى :

1 ـ التعرف على علاقة الإحساس بالقدم المسيطرة بدقة التصويب بكرة القدم

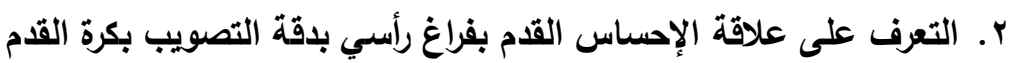

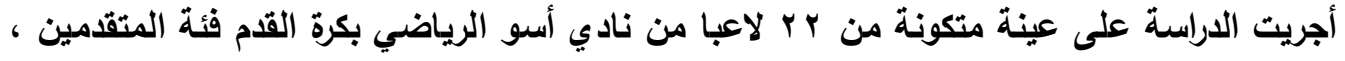
واستخدم الباحث اختبارين لقياس الحس - حركي واختبار ركل الكرة إلى الهذف (اختبار مهاري) لقياس

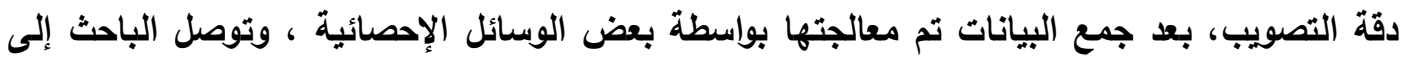

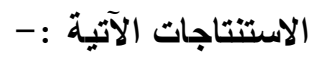

ا ـ وجود علاقة ارتباطيه معنوية بين الإحساس بالقدم المسيطرة ودقة التصويب بكرة القلم.

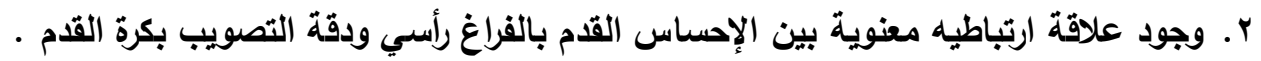
على ضوء الاستتتاجات يوصي الباحث بما يأتي : 1. زيادة اهتمام المدربين بلمج تمارين المهارات الأساسية بالقدرات الإدراك الدس - مركي وخصوصا مهارة التصويب 


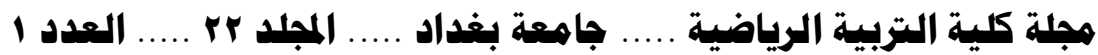

r. إجراء دراسة مشابهة للفئات العمرية (الناشئين والشباب) لتطوير الإدراك الحس - حركي وتأثثر في الأداء المهاري في كرة القدم .

r. إجراء دراسة مشابهة على الألعاب الأخرى (الفردية والجماعية) لتطوير المهارات الأساسية والإدراك الحس - حركي .

\section{$\underline{\text { Abstract }}$}

Relationship of Some Sense Knowing Ability Accuracy Motive of Scoring for Football Players of ASO CLUB at the Advanced Levels

Purpose of the Research :

Knowing the relation to the space of head and succeeded foot sense with scoring accuracy for football .

The study was carried out on one sample of ASO CLUB PLAYERS, whom were (22) football players the researcher used two tests of sense knowledge, motive and test of ball kicking to the goal for standard accuracy of scoring (goal recording), after collecting information they were solved from some statistics reasons and researcher has obtained some

Results, which were :

Taking into consideration of the coaches to train on specialty fall all skills from the basic skills from football .

Performing a similar study for young to develop of sense knowing movement and effect on having skill from football and their with the motive sense knowing abilities .

Carrying out a similar study on other games (single and team games) to develop basic skills and sense knowing _ motive .

Under the light of results, the researcher had these recommends :

Caring trainers more about merging the training of basic skills with sense knowing abilities _ movable and especially kicking skills .

Similar study procedure for age levels (children and young) for developing sense knowing _ movable and its influence on the ability skills in football .

Similar study procedure for other games (single and team games) for developing basic skills and knowing sense _ movable . 


\section{البـاب الأول}

\section{ا - التعريف بالبمث \\ 1-1 الاقدمة وأهمية البحثي}

إن الارتقاء بالمستوى الرياضي في كافة الأنشطة الرياضية يتوقف الى حد كبير على اكتمال الحالة

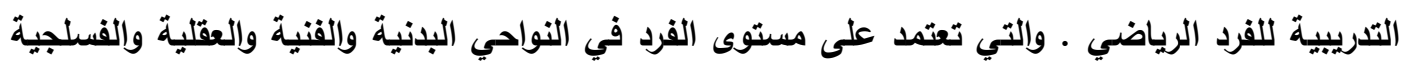

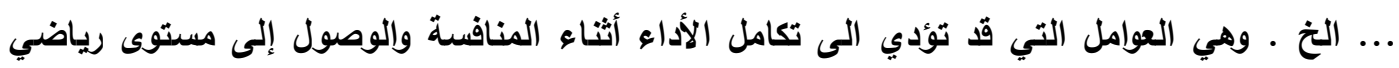
عالي

وفي الفترة الزمنية الأخيرة أصبح التدريب الرياضي في نتائجه معتمدا على العوامل والمتطلبات

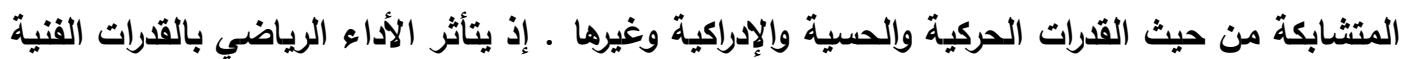
للرياضيين وخصائصهم البدنية وظروف اللعب لكل لاعب على حدة والفريق ككل .

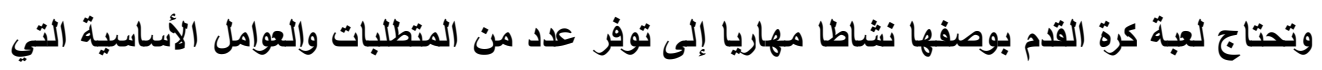

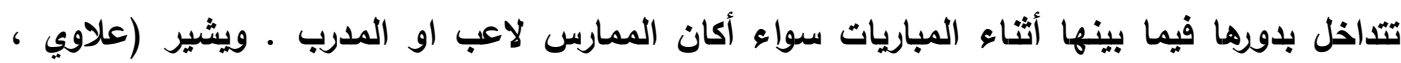
9 q 9 مثلا قرا من القوة لإيصالها الى الههف" ـ وتعد التصويب إحدى المهارات الأساسية مهمة في لعبة كرة القدم وهي النهاية الطبيعية لعملية الهجوم وهي فعالية هجومية يقوم بها اللاعب بمفرده في محاولة

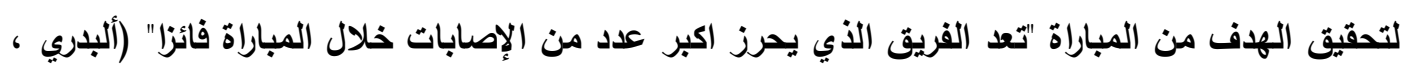
• ( $)$ ( $19 \wedge \mathrm{V}$

وتأتي أهمية البحث لاراسته علاقة بعض قدرات الإدراك الحس ـ حركي بدقة التصويب ، لكونها

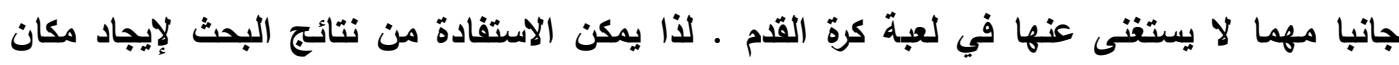
الضعف في الجانب المذكور عند لاعبي نادي أسو الرياضي ، واطلاع السادة المعنيين فيه بهذه النتائج بغية الوقف على ايجابيات النادي وسلبياته ومعالجته .

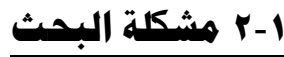

تعاني كرة القدم في نادي أسو الرياضي من المعوقات وإلمشاكل التي حالت دون تطورها والارتقاء بها ، منها غياب التخطيط العلمي المبرمج وعدم أتباع الوسائل العملية والعديثة في العملية التدريبية . ولعل النتائج التي حصل عليها النادي في المشاركات الاخلية والخارجية لمحافظة خير دليل على ذلك . . 


\section{هبلة كلية التربية الرياضية .... جاهعة بغداد ..... المبلد rr..... العدد I}

واعتمادا على الخبرة المتوافر للباحث باعتباره لاعبا سابقا ومدريا للعبة فقد لاحظ ان هناك تداخلا بين العمليات التدريبية والفسلجية والنفسية والتي تثترك فيما بينها في هذا المجال فقد تحتم عليه تحديد

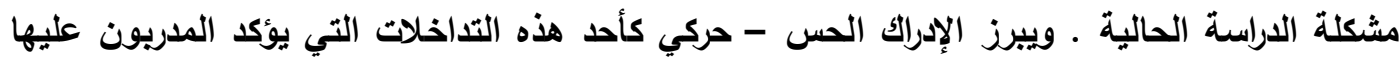

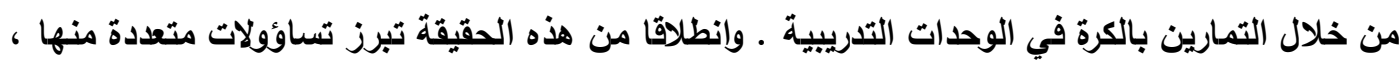

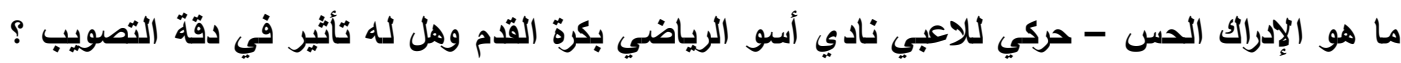
بغية وضع الأسس العلمية في أصول تدريب اللعبة ويما تخدم تطورها وصولا لتحقيق مستوى عال للاعبين وأدائهم لانجاز أفضل النتائج اعتمادا على أسلوب علمي صحيح .

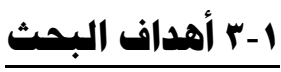

يرهف البحث الحالي الى التعرف على :

1. العلاقة بين الإحساس بالقدم المسيطرة ودقة التصويب بكرة القدم .

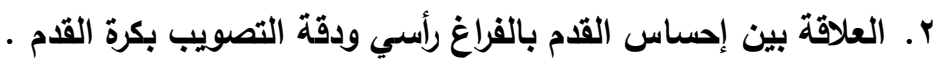

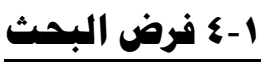

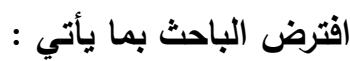
ا ـ وجود علاقة ذات إحصائية بين الإحساس بالقدم المسيطرة ودقة التصويب بكرة القدم .

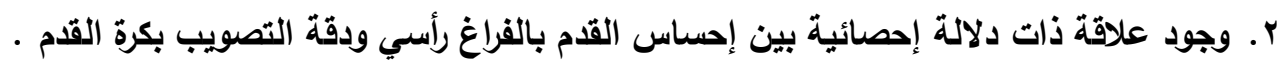

\section{1-0 هجالات البحث}

1- ه-1 المجال البشري : عينة من لاعبوا نادي أسو الرياضي - فئة المتقدمين البالغ عددهم (Y (Y)

لاعبا

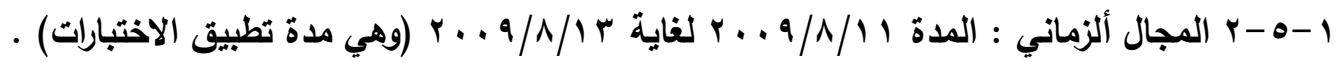
1-ه - المجال المكاني : ملعب نادي أسو الرياضي بكرة القدم (أربيل) • 
ه الإدراك الحس - حركي (Singer , 1976.299) بأنه "عبارة عن إدراك الفرد لأوضاع وحركات

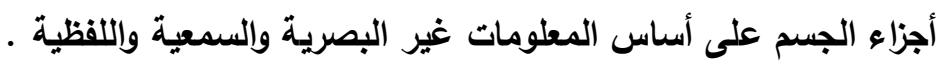

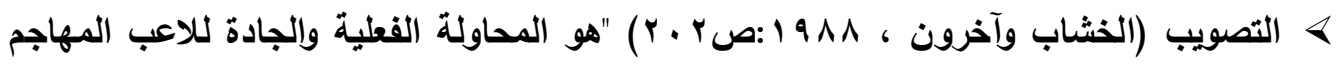

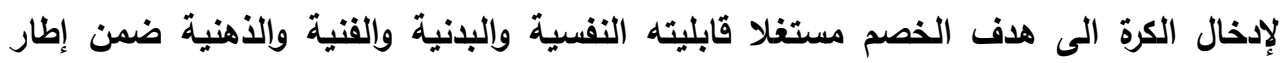

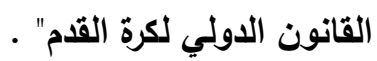

\section{البــاب الثانـي \\ r r الدراسات النظرية والدراسات المشابهة

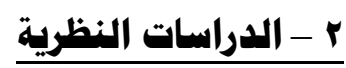 \\ r-1 - أهمية الإدراك الصس - حركي في المجال الرياضي}

يلعب الإدراك الحس - حركي دورا مهما في حل المشكلات المهارية التي تواجهه اللاعب أثناء التدريب والمنافسة فنواحي النشاط الرياضي في صورتها المتعددة وخططها ذات الطبيعة المختلفة تحتاج من الراضي ان إدراكا دائما لعناصر الموقف الذي يواجهه حتى يستطيع التغلب على الظروف المتغيرة في الملعب ، لذا يجب الاهتمام بقياس الإدراك الحس - حركي وتقويمه نظرا لاوره المهم في الإدرالك الحركي

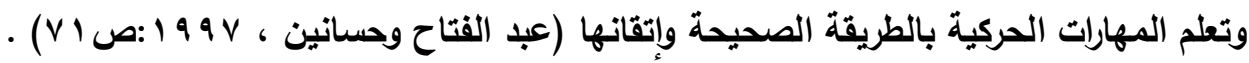

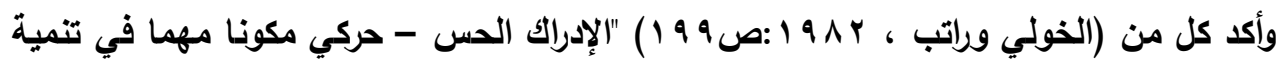
وتطور الجسم فعندما يمتلك الفرد مهارات إدراكية بمستوى جيد فنلك يعني نمو الجهاز العصبي الذي هني

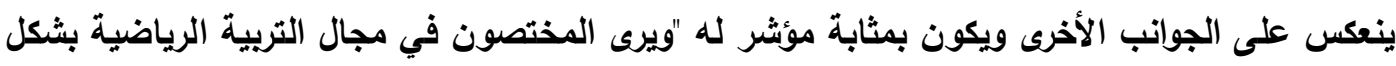
عام ايجابية بين الأداء في بعض الاختبارات الخاصة في الإدراك الحس - حركي والأداء في اختبارات

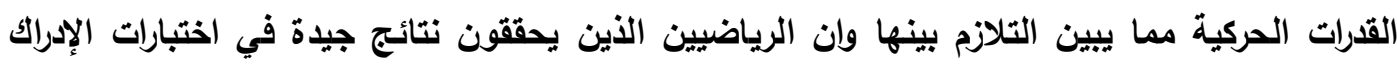

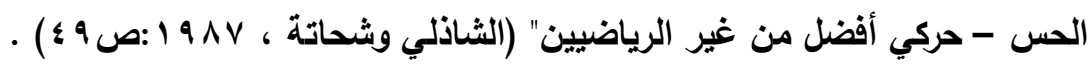

r r-l r المهارات الأساسية في كرة القدم 
تعد المهارات من العناصر الرئيسة والأساسية التي يجب ان يمتلكها لاعب كرة القدم إذ تكون في

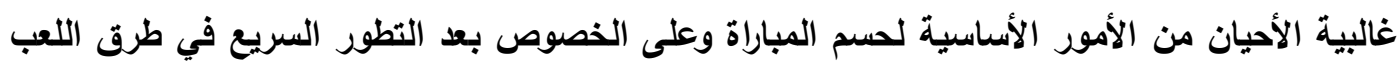

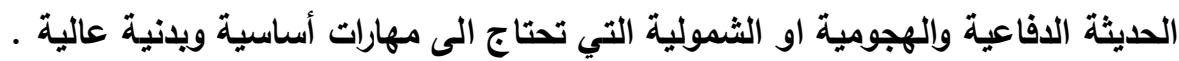

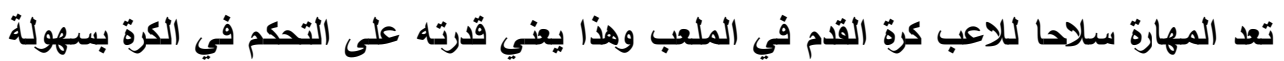
ويدون مجهود زائد فضلا عن أنها تساعد التلاعب في التظلب على أكثر الخصوم كما أنها تخلق لحظات

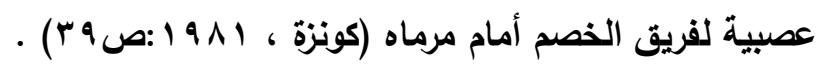

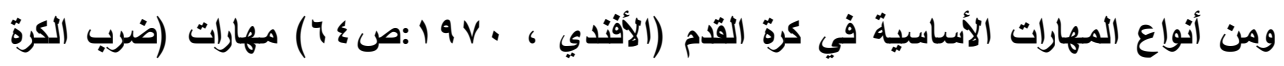

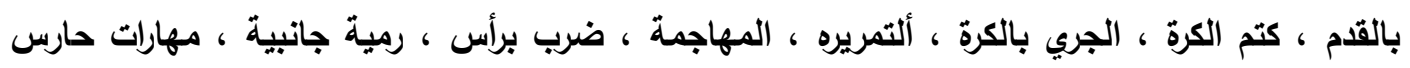

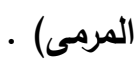

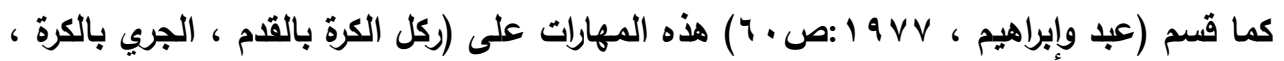

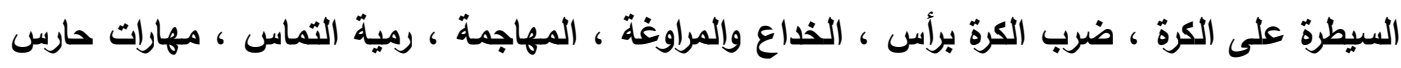

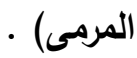

واستخدم الباحث في بحثه مهارة التصويب .

\section{r-r-r}

يعد التصويب من أهم المهارات الفنية التي يجب ان يتقتها لاعب كرة القدم وهي إحدى المهارات

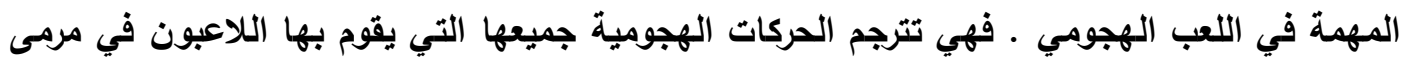

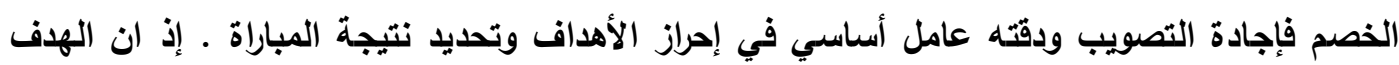

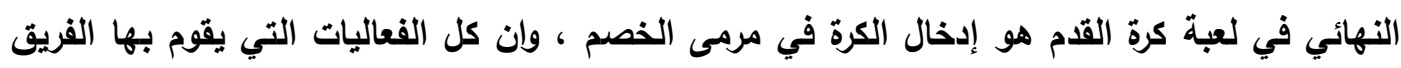
يجب ان تخدم هذا الهـف لتسجيل اكبر عدد من الأهداف على الرغم من المقاومة المنظمة التي يبليها

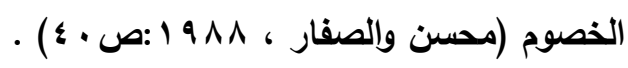

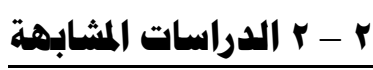
م 1999 م-r (الإدراك المسي عضلي وعلاقتهه ببعض المهارات الأساسية بكرة اليد).

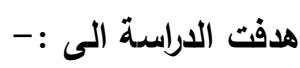




\section{هبلة كلية التربية الرياضية .... جاهعة بغداد ..... المبلد r. ..... العدد I}

التعرف على الإدراك الحسي عضلي للأراع المستخدمة بعينة البحث وعلاقته ببعض المهارات

الأساسية بكرة اليل .

بلغ حجم العينة (r Y ) لاعبا من نادي الفتوة الرياضي للمتقدمين بكرة اليد ، وأسفرت نتائج

البحث عن وجود علاقة ذات دلالة معنوية بين الإدراك الدس عضلي للأراع الرامية ويبن المهارات

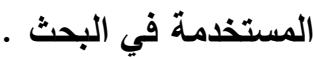

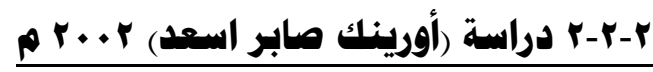
(بعض قدرات الإدراك الصس - حركي وعلاقتها بعناصر الالياقة البدنية الخاصة وبعض المهارات

\section{الهجموهية بكرة السلة) .}

التعرف على العلاقة الموجودة بين بعض القدرات الإدراك الحس - حركي وعناصر اللياقة البلنية

الخاصة في كرة السلة

التعرف على العلاقة الموجودة بين بعض القدرات الإدراك الحس - حركي ويعض المهارات

الهجومية في كرة السلة .

تم إجراء الدراسة على عينة قوامها (Yr) لاعبة يمثلون نادي أكاد وسنحاريب الرياضي بكرة

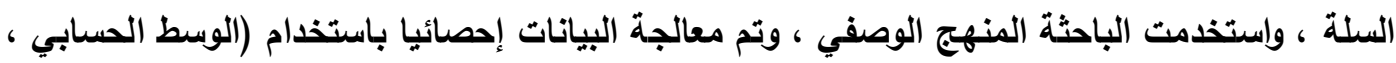

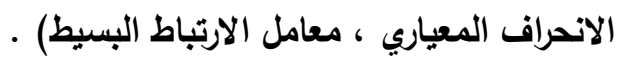

\section{البــاب الثالهـثـ}

r - هنهج البمث وإجراءاته الميدانية

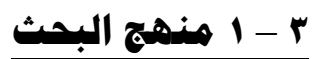

استخدم الباحث المنهج الوصفي بأسلوب ألارتباطي لملائمته وطبيعة المشكلة . 


\section{هجلة كلية التربية الرياضية .... جاهعة بغداد ..... المبلد r ..... العدد I}

تم اختيار لاعبي نادي أسو الرياضي بكرة القدم من فئة المتقدمين بطريقة عمديه والبالغ عددهم

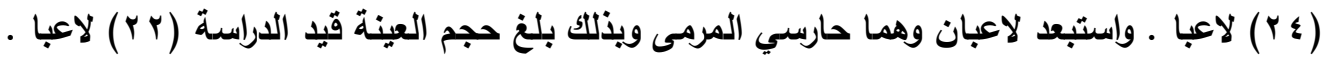

\section{r r r أدوات ووسائل جمع المعلومات}

الأدوات شملت : - ادوات

> (ملعب كرة القدم ، كرة القدم ، شريط القياس ، قطعة قماش ، حائط مدرج)

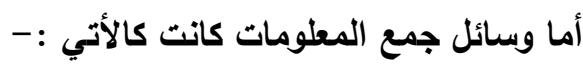
ه (المصادر العربية والأجنبية ، استمارة استبيان ، أراء المختصين ، الوسائل الإحصائية) .

\section{r - ـ تصديد قدرات الإدراك الهس - حركي والاختبارات الملائمئة}

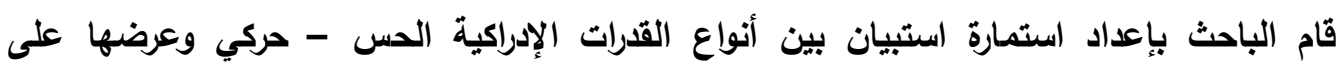
مجموعة من المختصين(*) في مجال اللعبة والتعلم الحركي (ملحق 1) ، بهدف تحديد القدرات الأكثر

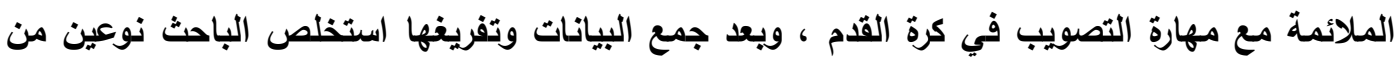

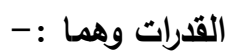

1. الإحساس بالقدم المسيطرة .

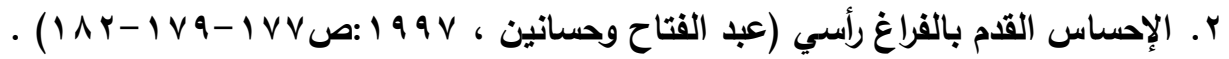
ثم قام الباحث بتصميم استمارة أخرى (ملحق ب ) يهاف معرفة مدى إمكانية استخدام الاختبارين التي تم اختيارها من المصادر المتعلقة لقياس القدرات المرشحة للاراسة ويعد جمع الاستمارات ابدوا

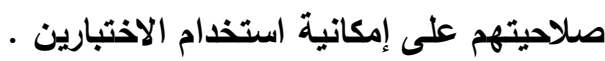

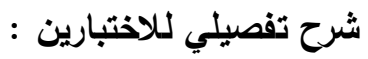

الاختبار الأول : الإحساس بالقدم المسيطرة

* يكون الخبراء والمختصين من :

ه أ.م.د. صباح مصطقى - كلية التربية الرياضية / جامعة بغداد .

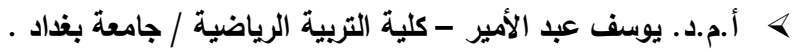

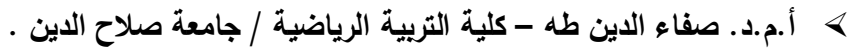
> أ.م.د. ثيلام يونس علاوي - كلية التربية الرياضية / جامعة الموصل .

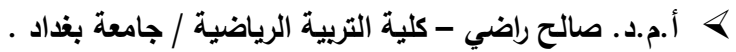




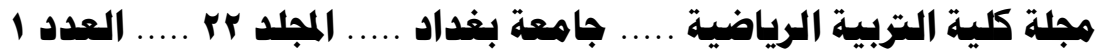

الهذف من الاختبار : قياس قدرة القدم المسيطرة على الإحساس بالمسافة الجانبية . الأدوات المستخدمة : شريط القياس - قطعة قماش لعصب العينين .

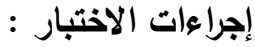

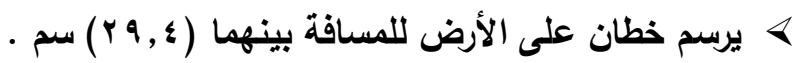
> تقف المختبرة بحيث تكون قدمها المسيطرة موازية للخط الأيسر ، أي ان تكون قدمها اليمنى

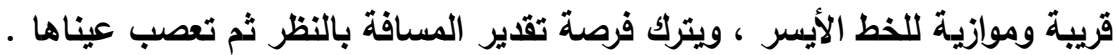
> تقوم اللاعبة وهي معصوية العينين بنقل قدمها اليمنى جانبا الى الخط الثاني (الخط الأيمن)

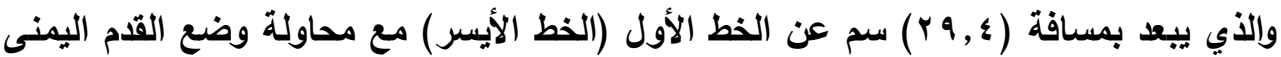
على الحافة الخارجية للخط الثاني كما موضح في الثكل .
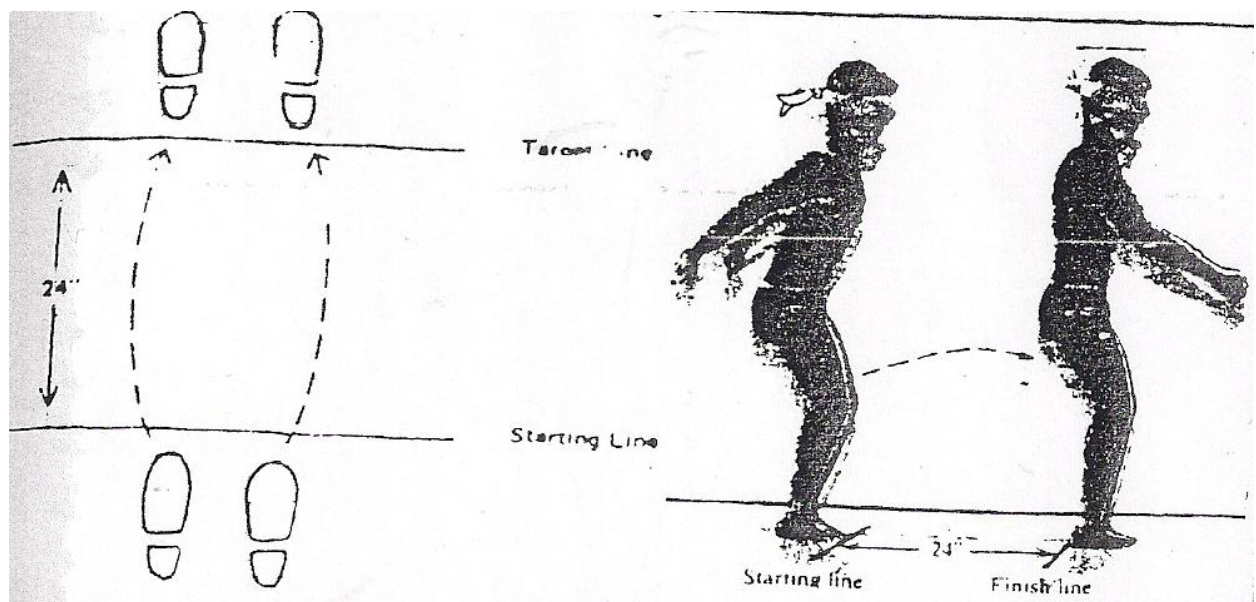

طريقة التسجيل

> تسحب المسافة من القدم حنى الحافة الخارجية للخط الثاني . < تتاح للاعبة ثلاثة محاولات بحيث تسجل لها مجموع المحاولات الثلاث والتي تمثل مجموعة

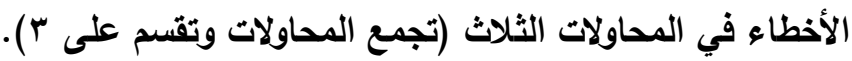
> تلد دقة نقل إحدى القدمين جانبا للمسافة المحدة مسبقا على ارتفاع مستوى الإحساس شريطة

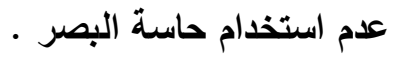

الاختبار الثاني : إحساس القدم بالفراغ رأسي $(\uparrow \wedge 1)$ 


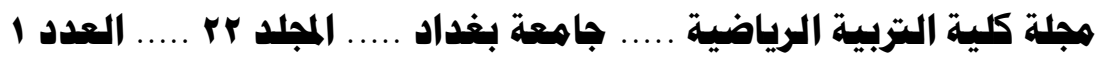

الهدف من الاختبار : قياس الإحساس بحركة رفع القدم عموديا (إلى الأعلى) عند ثني مفصل الركبة .

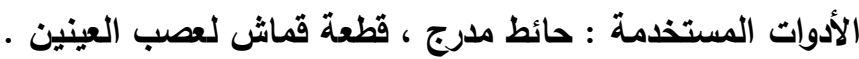

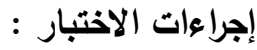

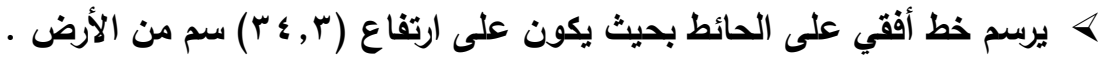

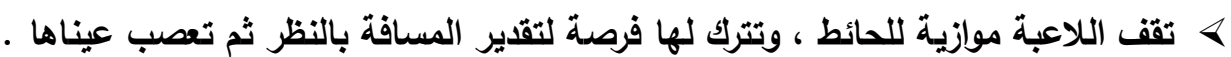
> تحاول اللاعبة رفع قمها المسيطرة وإلمجاورة للحائط الى مستوى الخط المرسوم على الحائط ، ويكون ذلك عن طريق ثني ركبة الرجل المسيطرة ، كما موضح في الثكل التالي : -

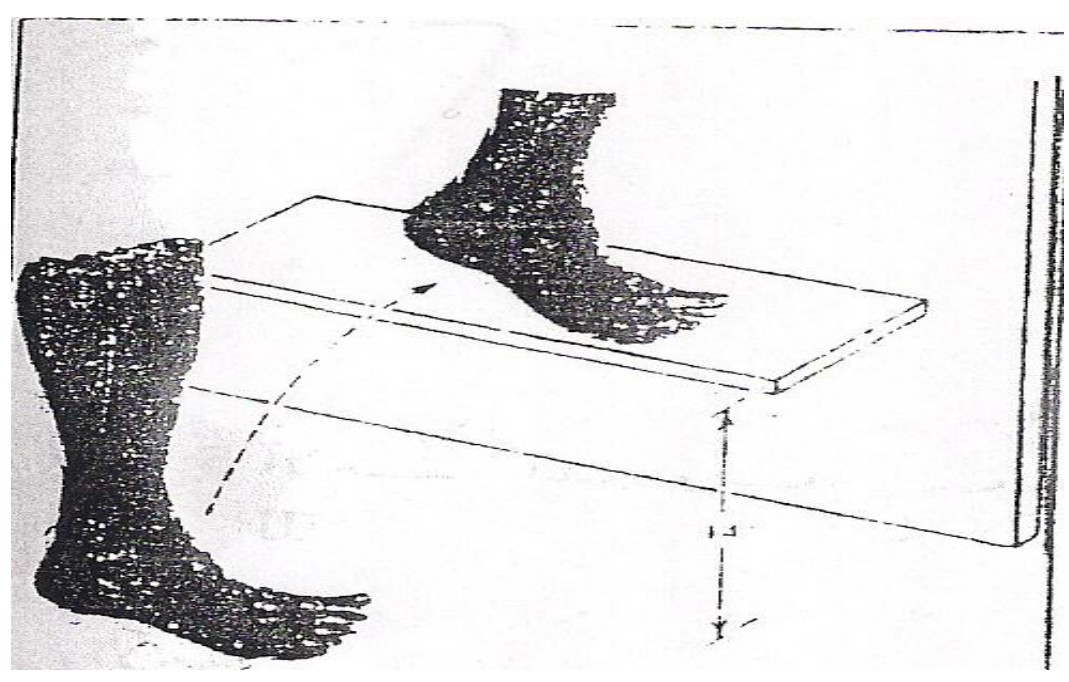

طريقة التسجيل ه يتم تسجيل المسافة بين القدم والخط المرسوم على الحائط بقرب ( الج, • ) سم . ه تتاح للاعبة محاولتان وسيجل لها مجموعهما .

r - r تهديد الاختبار المهاري (التصويب)

قام الباحث بإعداد استمارة استبيان أخرى يتبين اختبار مهارة التصويب (ملحق r) وثم عرضها على مجموعة من المختصين في مجال كرة القدم ومجالات أخرى (مختصي اختبارات الأداء الحس - 
حركي) أنفسه ، وطلب من المختصين تحديد الأنسب الاختبار بين أريعة اختبارات المرشحة وفي حالة عدم صلاحية الاختبار يرجى بيان البديل ، ويعد جمع البيانات توصل الباحث الى الاختبار الأتي : -

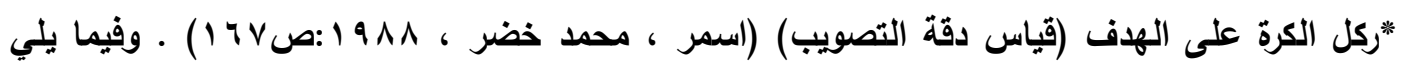

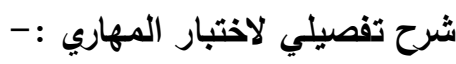

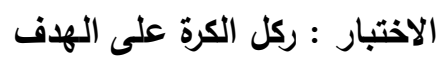

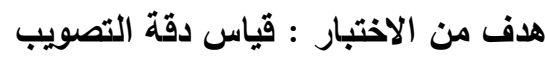
الأدوات الأزمة : ملعب كرة قدم ، كرات قدم عدد ( · 1) ، شريط لتعين منطقة التصويب للاختبار ، شريط القياس الاسوات إجراءات الاختبار : توضع (• (1) كرات قدم في أماكن مختلفة على خط وداخل منطقة الجزاء، وكما موضح

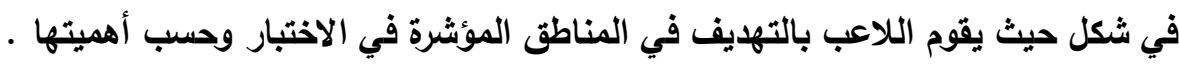

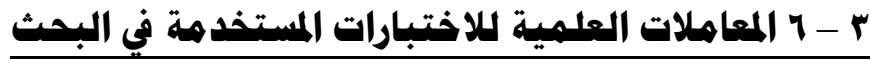

من اجل التأكد من مدى ملائمة الاختبارات المرشحة لعينة البحث وإمكانية استخدامها لقياس الإدراك الحس - حركي قيد الاراسة والاختبار المهاري ، قام الباحث بتحقيق من المعاملات العلمية

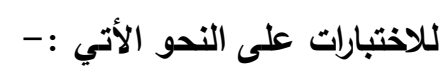

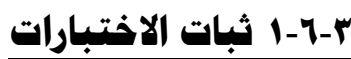

تم استخراج ثبات الاختبارات (الإدراك لحس - حركية و المهارية) بطريقة إعادة الاختبار ، إذ إذات

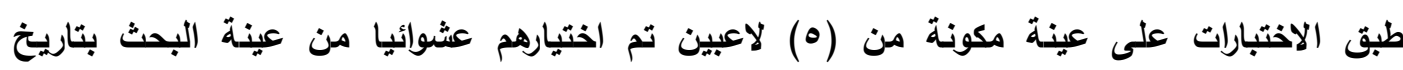

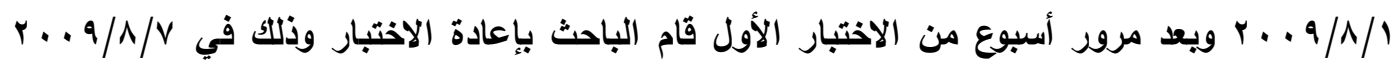
ويعد استخراج الارتباط بين الاختبارين أظهرت بان الاختبارات تتمتع بلرجة عالية من الأبن الثبات كما كوضح

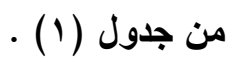

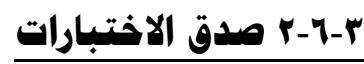

رغم حصول الباحث على صدق الاختبارات المستخدمة في البحث عن طريق استمارة الاستبيان

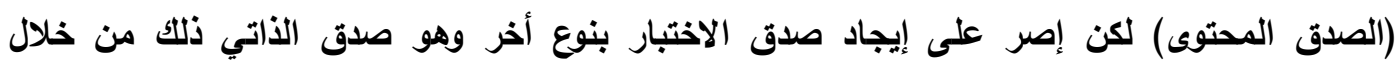

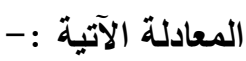




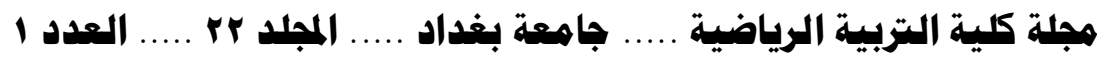

الصدق الاتي = / ثبات الاختبار وكما موضح في جدول (1) ·

r-r-r هوضوعية الاختبارات

تم التأكد من موضوعية الاختبارات من خلال عدم تلخل الباحث من تسجيل البيانات بل كانت

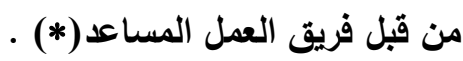

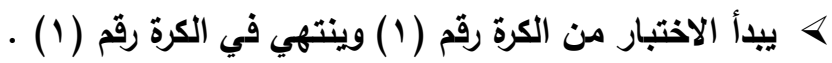

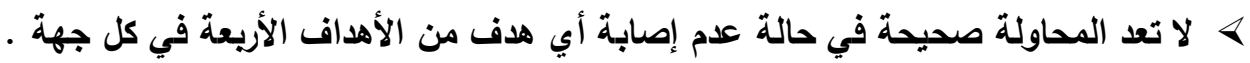

\section{| r - r التجربة الاستطلاعية}

قام الباحث بإجراء التجربة الاستطلاعية بتاريخ 9/1/9 . . . . على لاعبي نادي أسو الرياضي في كرة القدم وإختيروا بشكل عشوائي من مجتمع البحث نفسه ويلغ عددهم ( ( ) لاعبين وعدم استبعاده من التجرية الرئيسية ، وكان الهذف من التجرية ، هو الوصول إلى أفضل الوسائل التي تخدم البحث : \& التعرف على مدى استجابة العينة لأداء الاختبارات . ه التعرف على المشاكل والصعويات التي تواجه الباحث وفريق العمل أثناء تنفيذ الاختبارات . ه الوصول إلى معرفة البدائل الللازمة قبل تنفيذ التجرية الرئيسية .

\section{r - r التجربة الرئيسية}

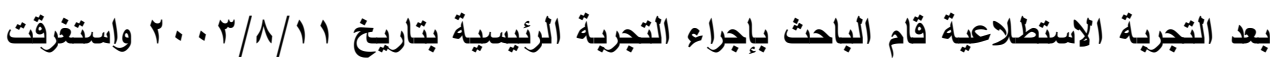
مدة إجراء الاختبارات يومين كالأتي : اليوم الأول مخصصا للاختبارات المهارية في كرة القدم ، اليوم

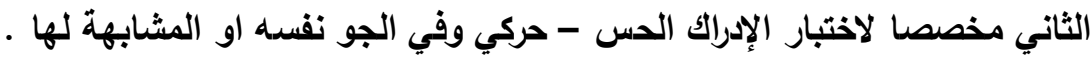

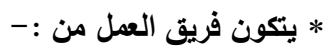

> ا.م. سامان حمد سليمان (ماجستير) - كلية التربية الرياضية - جامعة صلاح الاين .

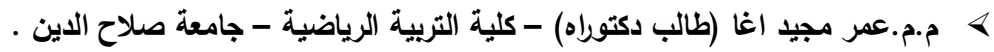

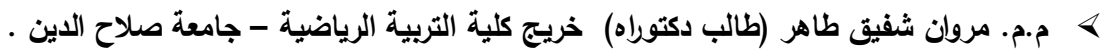

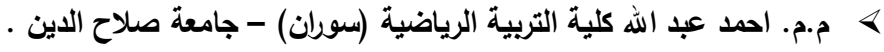


هبلة كلية التربية الرياضية .... جاهعة بغداد ..... المبلد r ..... العدد ا....

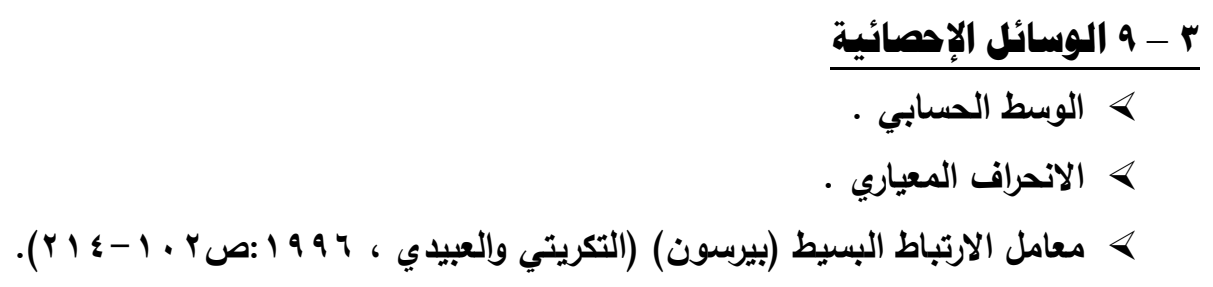

جدول (1)

يبين معامل الارتباط بين الاختبارين الأول والثاني وصدق الاختبارات المستخدمة وقيمة (R) المحتسبة

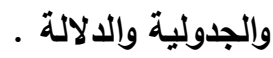

\begin{tabular}{|c|c|c|c|c|}
\hline الالالة & $\begin{array}{c}\text { * R } \\
\text { الجدولية }\end{array}$ & معامل الصدق & بين الاختبارين الارتباط & الاختبارات المستخدمة \\
\hline غير معنوي & \multirow{3}{*}{$\cdot, \varepsilon r r$} & $\cdot, 9 \leqslant$ & $\cdot, \wedge \wedge$ & الإحساس بالقدم المسيطرة \\
\hline غير معنوي & & $\cdot, 9 \vee$ & $\cdot, 9$. & الإحساس القدم بالفراغ \\
\hline غير معنوي & & $\cdot, \wedge \vee$ & $\cdot, v$ & ركل الكرة إلى الهدف \\
\hline
\end{tabular}

*تحت درجة حرية (r) ونسبة خطأ (ه . , •) .

يتبين من الجدول ( ) بأنه معامل الارتباط بين الاختبارين الأول والثاني عالية جدا وأيضا صدق الثق

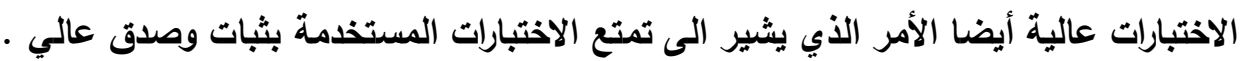

\section{البـاب الرابـع}

\section{ع - عرض النتائج ومناقشتها \\ ع - 1 عرض النتائج وتهليلها وهناقشتها}




\section{هجلة كلية التربية الرياضية .... جاهعة بغداد ..... المبلد r ..... العدد I. I}

لتحقق مدى صحة فرضيتا البحث الذي تم وضعها ومن اجل عرض البيانات الإحصائية التي

$$
\text { حصل عليها الباحث تم وضعها في إعداد الجدول الأتي :- }
$$

\section{ع-1-1 عرض نتائج علاقة الارتباط بين بعض قدرات الإدراك الصس - حركي ودقة التصويب}

\section{جدول (r)}

يبين علاقة الارتباط ين بعض قدرات الإدراك الدس - حركية وإلدقة التصويب بكرة القدم

\begin{tabular}{|c|c|}
\hline الاختبار المهاري & \multirow{2}{*}{ قرات الإدراك الحس - حركي } \\
\hline دقة التصويب & \\
\hline •, & الإحساس بالقدم \\
\hline$\cdot, \curlyvee \wedge *$ & الإحساس القدم بالفراغ الرأسي \\
\hline
\end{tabular}

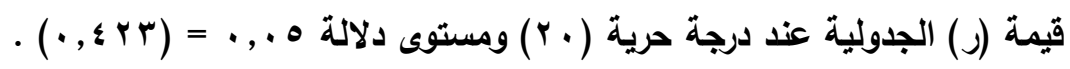

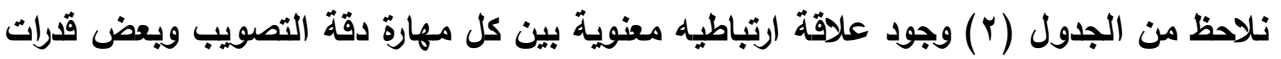

الإدراك الحس - حركي (الإحساس بالقدم المسيطرة والإحساس القدم بالفراغ الرأسي) إذ بلغت قيم الجيم معامل

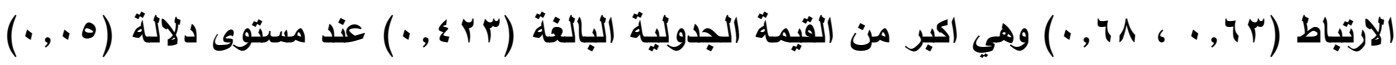

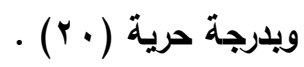

يتضح من الجدول (Y) ان العلاقة ين كل من الإحساس بالقدم المسيطرة والإحساس القدم

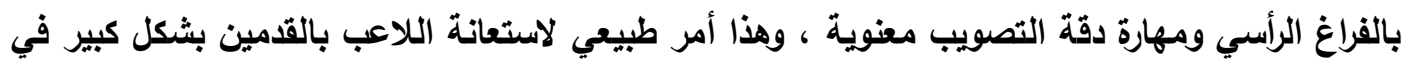

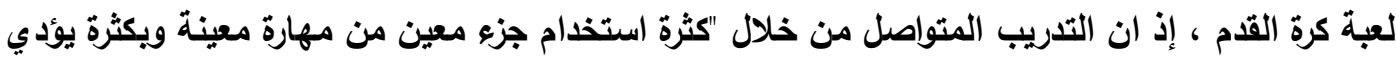

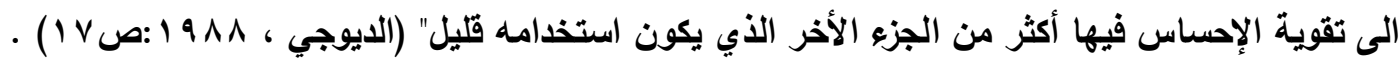

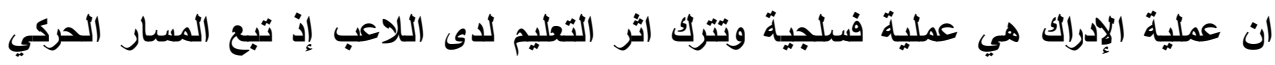
الصحيح ، لذا يركز اغلب المدربين على التعلم ويشمل في مضمونه الإدراك منذ الصغر للوصول اللى الصى مستويات متقدة ، وهذا يعني انه كلما ارتفعت نسبة التعلم الى نسبة الإدراك كلما ارتفع مستوى الأداء 


\section{هجلة كلية التربية الرياضية .... جاهعة بغداد .... المبلد r ..... العدد I}

المهاري ويذكر (Singer , 1976:p12) "بان الإدراك الحس - حركي يرتبط بسرعة التطلم" لذا فان عملية التأكيد على التعليم للوصول الى مرحلة متقدمة من المستوى المهاري الجيد يصاحبها تطور في الإدراك

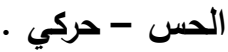

\section{البـاب الخاهـس}

0 - الاستنتاجات والتوصيات

0 - 1 الاستنتاجات

I. وجود علاقة ارتباطيه معنوية بين الإحساس بالقدم المسيطرة ودقة التصويب بكرة القدم . r. r. وجود علاقة ارتباطيه معنوية ين الإحساس القدم بالفراغ الرأسي ودقة التصويب بكرة القدام .

I. زيادة اهتما العدريين بدمج تمارين المهارات الأساسية بالقدرات الإدراك الدس - حركي وخصوصا مهارة التصويب . بان. r. إجراء دراسة مشابهة للفئات العمرة ( الناثشئين والثباب) لتطوير الإدراك الحس -حركي وتأثير في

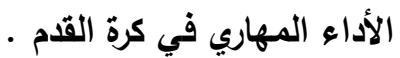
r. إجراء دراسة مشابهة على الألعاب الأخرى (الفردية والجماعية) لتطوير المهارات الأساسية

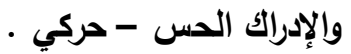

\section{المسادر العربيسة والأجنبيسية}

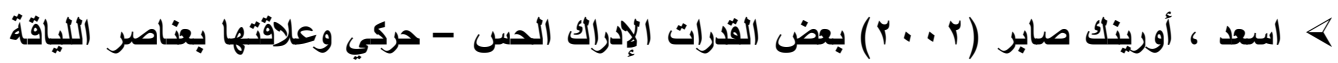
البلنية الخاصة ويعض المهارات الهجومية بكرة السلة ، رسالة ماجستير غير منشورة ، كلية الإلية

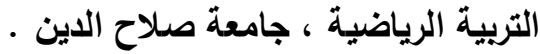


ه اسعد ، محمد خضر (1991) اثر أسلوب المنافسات والتغذية الراجعة المقارنة في الرضي

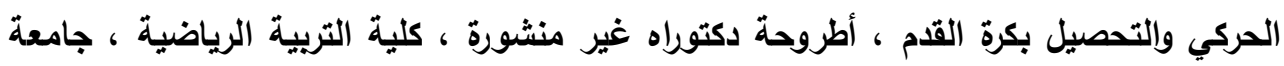
الموصل .

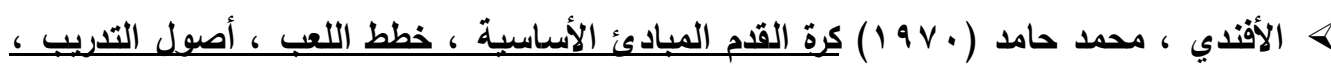
قانون اللعب التحكيم؛ عالم الكتب ، القاهرة .

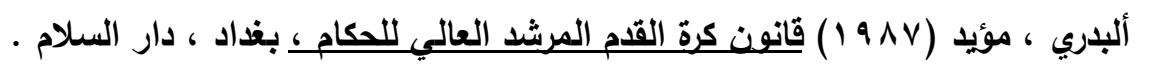
التكريتي ، وديع ياسين والعبيدي ، محمد حسن (997) ، (99) ، التطبيقات الإحصائية في المجال

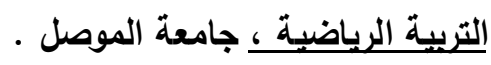

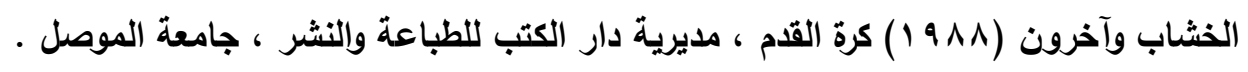

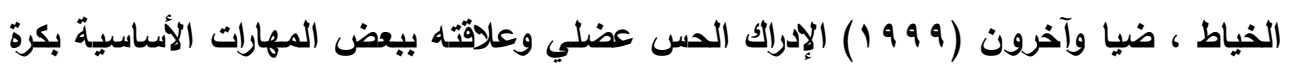

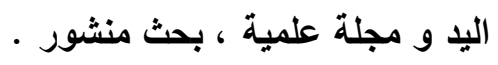

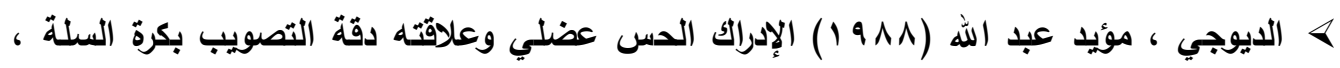

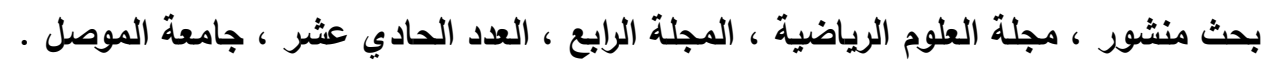

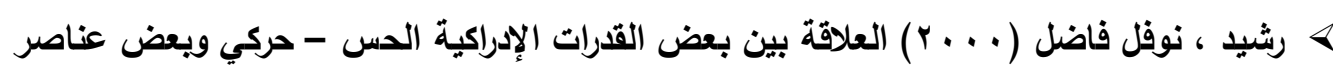
اللياقة البدنية والصفات المهارية لاى لاعبي كرة القدم ، رسالة ماجستير غير منشورة ، جامعة الفيل الموصل \& الثاذلي ، احمد فؤاد وشحاتة ، احمد إبراهيم (9 1 1 ) الأهمية النسبية لبعض أنواع الإحساسات المرتبطة بالأداء المهاري للاعب الجمباز تحت سن (1 المأدة سنة ، بحث منشور في المؤتمر

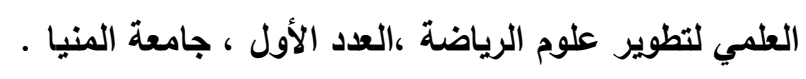

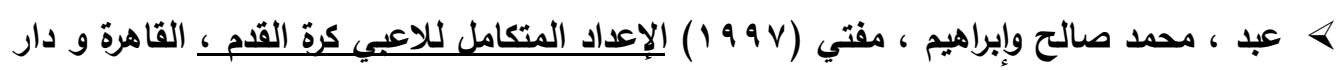

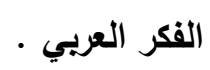
> عبد الفتاح ، أبو العلا وحسانين ، محمد صبحي (99VV) فيبولوحيا وموفولوحيا وطرق

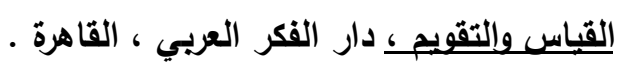

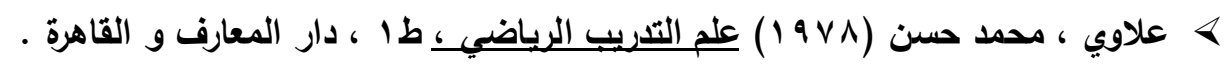

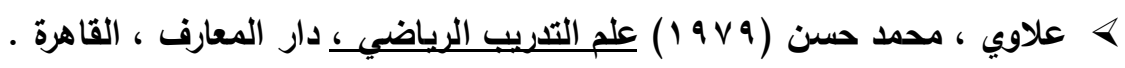




\section{هجلة كلية التربية الرياضية .... جاهعة بغداد ..... المبلد r ..... العدد I. I}

ه كونزة ، الفريد (911) كرة القدمـ، ترجمة و ماهر ألبياتي وسليمان علي حسن و جامعة

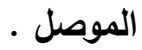

ه محسن و ثامر والصفار ، سامي (911 1 ) أصبول التّريب في كرة القدم ، مطبعة جامعة الموصل

Singer (1976) R.N motor Learning and human performance . Y. Macmillan .

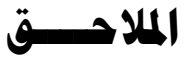

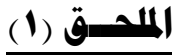 \\ استمهارة استببيان}

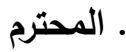

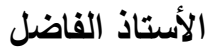

في النية إجراء البحث الموسوم (بعض القدرات الإدراك الدس - حركي وعلاقته بدقة التصويب في كرة

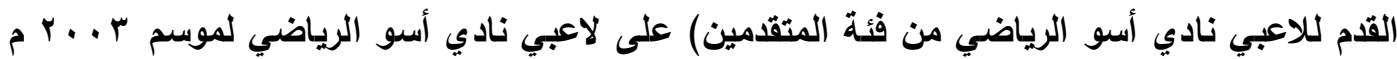
فئة المتقدمين ماعين ونظرا لما تتمتعون به من خبرة ودراية في هذا المجال لذا يرجى التفضل باختيار أهم القدرات من الإدراك

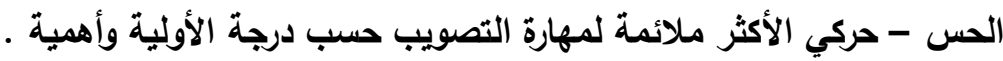
وتقبلوا فائق الثكر والاحترام

الباحث

\begin{tabular}{|c|c|c|c|c|c|c|c|c|}
\hline الملاحظات & 9 & $\bullet$ & $\varepsilon$ & $r$ & $r$ & 1 & الاختبارات الإدراك الحس - حركي & 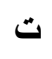 \\
\hline & & & & & & & الإحساس بمسافة الوثب & 1 \\
\hline & & & & & & & الإحساس برمي الكرة & r \\
\hline & & & & & & & الإحساس القدم بالفراغ الرأسي & $r$ \\
\hline
\end{tabular}




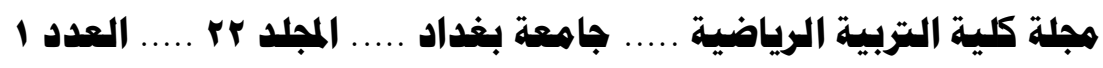

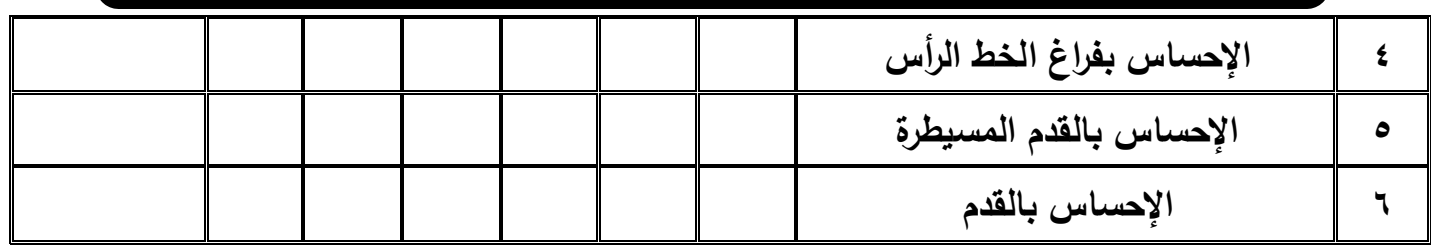

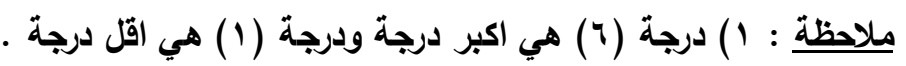

ץ تم توزيع كافة إجراءات الاختبارات الإدراك الحس - حركي الى المختصين مع استمارة استبيان .

الاختصاص

الارجة العلمية

الاسم والتوقيع

\section{(r) (I) \\ استمهارة استبيان}

الأستاذ الفاضل ...................................................................... المحترم

في النية إجراء البحث الموسوم (بعض القدرات الإدراك الحس - حركي وعلاقتّه بدقة التصويب في كرة

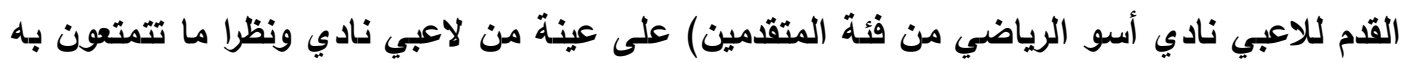

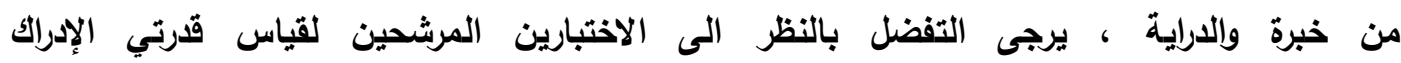
الحس - حركي · وبيان صلاحيتها او عدم صلاحيتها وفي حالة عدم صلاحيتها يرجى ذكر البديل

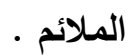

الباحث

\begin{tabular}{|c|c|c|}
\hline الاختبار & القدرات الإدراك الحس - حركي المرشحة & 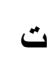 \\
\hline قدرة القام المسيطرة على الإحساس & الإحساس بالقدم المسيطرة & 1 \\
\hline قارة الإحساس بحركة رفع القدم عموديا & الإحساس القام بالفراغ الزأسي & r \\
\hline
\end{tabular}




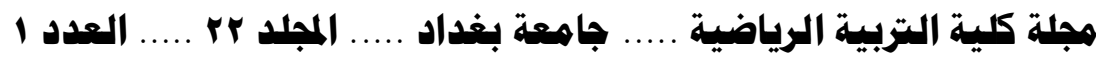

(الى الأعلى) عند ثني مفصل الركبة

ملاحظة : تم بيان تفاصيل الاختبارين على شكل ورقة المرفقة مع الاستبيان الذي تم عرضه على المختصين .

الاختصاص

الدرجة العلمية

الاسم والتوقيع

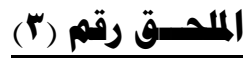 \\ استسمارة استببيان}

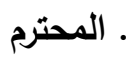

الأستاذ الفاضل

في النية إجراء البحث الموسوم (بعض القدرات الإدراك الدس - حركي وعلاقته بدقة التصويب في كرة

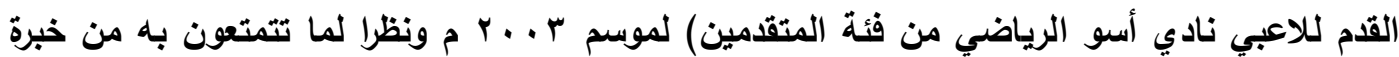
ودراية في هذا المجال يرجى التفضل بالاطلاع على الاختبارات المرشحة ومن ثم بيان رأيكم حول تحديد لاديد الأنسب الاختبار للفئة العمرية ، وفي حالة عدم صلاحيتها يرجى بيان الاختبار البديل .

الباحث

\begin{tabular}{|c|c|c|c|c|c|c|c|}
\hline البديل & صالحة & صالحة & التسجيل & مواصفات & الغرض من & الاختبار & المهارة \\
\hline & & & & & قياس دقة & ركل الكرة على هدف & التصويب \\
\hline
\end{tabular}




$$
\text { هبلة كلية التربية الرياضية .... جاهعة بغداد .... المجلد rr..... العدد ا }
$$

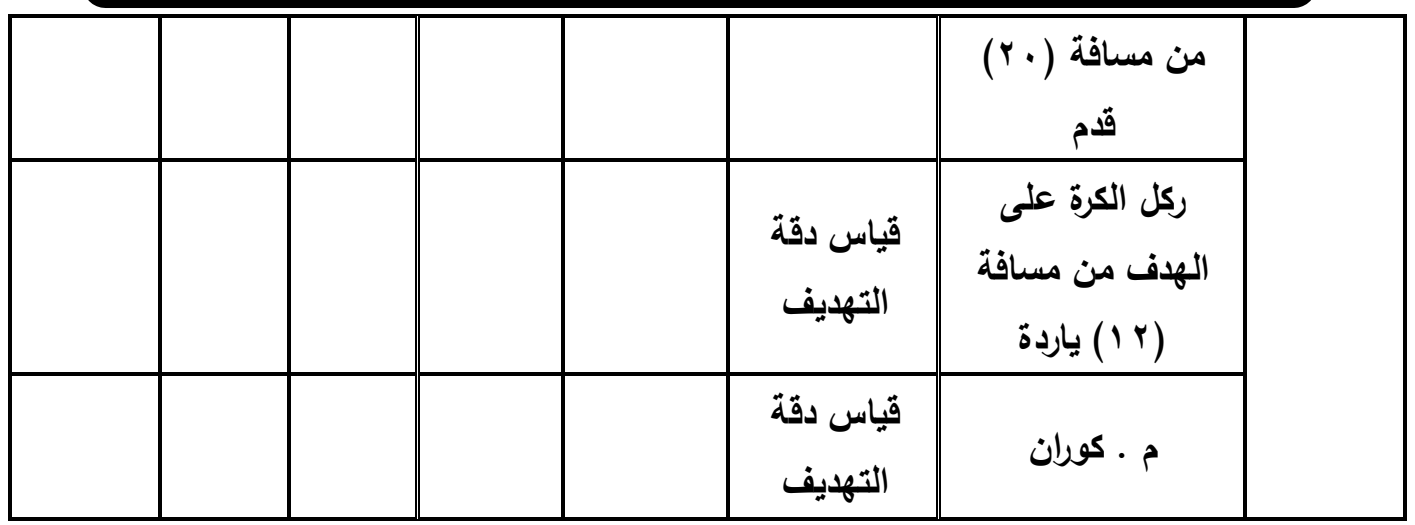

| الاختصاص

الارجة العلمية

الاسم والتوقيع 